EPJ Web of Conferences 41, 07001 (2013)

DOI: $10.1051 /$ epjconf/20134107001

(C) Owned by the authors, published by EDP Sciences, 2013

\title{
Photophysical processes of the spectroscopic RNA probe 2-(1-ethynylpyrene)-adenosine (PyA)
}

\author{
P. Trojanowski ${ }^{1}$, J. Plötner ${ }^{1}$, C. Grünewald ${ }^{2}$, M. Braun ${ }^{1}$, A. Reuss ${ }^{1}$, J. Engels $^{2}$ and J. Wachtveitl ${ }^{1}$ \\ ${ }^{1}$ Institut für Physikalische und Theoretische Chemie and ${ }^{2}$ Institut für Organische Chemie, Johann \\ Wolfgang Goethe-Universität, Max-von-Laue-Str. 7, 60438 Frankfurt am Main, Germany \\ E-Mail:wveitl@theochem.uni-frankfurt.de
}

\begin{abstract}
We examine the photoinduced excited state dynamics of pyrene modified adenosine, a versatile probe for folding and hybridization of ribonucleic acids. Measurements in different solvents revealed complex ultrafast dynamics, but high robustness since the overall fluorescence quantum yield $\left(\Phi_{\mathrm{f}}\right)$ is hardly affected. The result is a strong fluorescent RNA-probe whose spectral properties change in a defined way upon environmental changes.
\end{abstract}

\section{Introduction}

Due to its high fluorescence quantum efficiency and the sensitivity on its environment pyrenes are commonly used as fluorescent probes for/in biomolecular spectroscopy [1-3]. Pyrene modified adenosine (PyA, Fig. 1, A) represents a fluorophore label that can e.g. act as an universal RNA folding probe which only slightly disturbs the RNA structure. The pyrene moiety is thought to point out into the minor groove of RNA in an A-form helix [4]. Another favorable characteristic is the ability to form excimers upon close proximity of two PyAs of complementary strands in the minor groove [4]. Experiments revealed that PyA is also able to intercalate into RNA depending on the flanking bases in 5' direction of the modified base [5, 6]. A G-C base pair leads to the intercalation of PyA, whereas A-U base pairs do not show this behavior. To get a further understanding of the processes, the photophysical properties of PyA were investigated with femtosecond pump-probe absorption spectroscopy and time correlated single photon counting (TCSPC) [6, 7]. PyA showed the existence of a locally excited (LE) state with sequence dependent lifetimes around $400 \mathrm{ps}$ and presumably a relaxed S1 state with a lifetime around $2 \mathrm{~ns}$. These states differ only slightly in their energy and interconvert on a timescale of 3 ps [6]. The above mentioned excimer shows an additional lifetime of approximately $19 \mathrm{~ns}$ and a spectral red-shift of $50 \mathrm{~nm}$. Here we investigate the dependence of PyA on the solvent polarity. Combining these results with DFT calculations we are able to obtain a better understanding of the occurring processes. We observed a high sensitivity of PyA to its surroundings, but robustness of the high quantum yield. This makes PyA a suitable probe for more complex RNA systems. 


\section{Materials and Methods}

PyA synthesis is described in [4]. The PyA solutions were prepared by dilution of a PyA DMSO stock solution with the dried solvents. The end concentration of DMSO was approximately $2.5 \%$. No change in the absorption or fluorescence spectra in comparison to solutions without DMSO was observed. The pump-probe absorption spectroscopy setup was described in detail elsewhere [8]. The samples were excited with the frequency doubled pulses of a CLARK CPA 2001 (Clark-MXR, Dexter, MI), providing excitation laser pulses of $150 \mathrm{~nJ}$ at $388 \mathrm{~nm}$ with a pulse duration of $200 \mathrm{fs}$. UV-vis spectra recorded before and after the measurements revealed only slight photodegradation during the measurements. For the data analysis the solvent background along with the coherent artifact was subtracted and corrected for group velocity dispersion [9]. The quantitative analysis was performed employing a kinetic model describing the data as a sum of exponential decays [7].
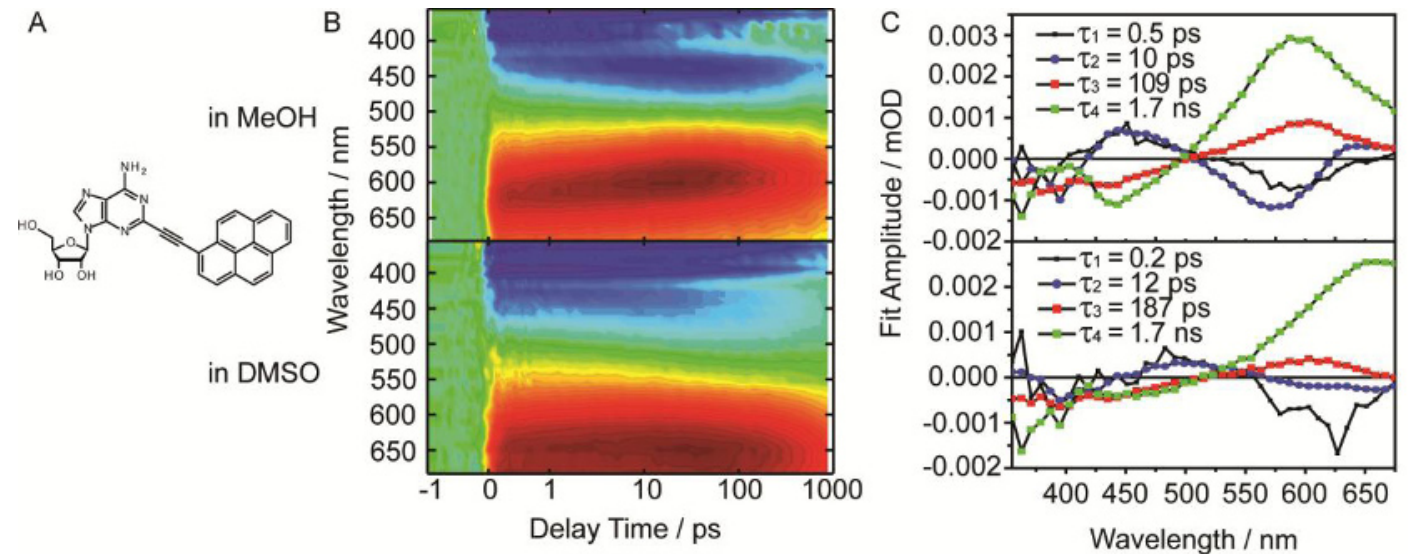

Figure 1: Structure of 2-(1-ethynylpyrene)-adenosine (PyA) (A). Transient absorption difference spectra (B) and decay associated spectra (C) of PyA in $\mathrm{MeOH}$ (top) and DMSO (bottom), $\tau_{1}-\tau_{4}$ denote time constants (in ps) derived from the global fitting procedure.

\section{Results}

The transient absorption spectra (Fig. 1, B) show the evolution of the electronic states after excitation of PyA in MeOH (upper panel) and DMSO (lower panel) over a time range of $1 \mathrm{~ns}$ in the spectral range from 355 to $675 \mathrm{~nm}$. Those spectra were taken for a total of 4 protic and 5 aprotic solvents (data not shown) covering a broad range of polarity. The spectral characteristics in $\mathrm{MeOH}$ are similar to those reported for PyA introduced in RNA strands dissolved in buffer [7] and of a silyl-protected derivate of $\mathrm{PyA}$ in $\mathrm{MeOH}$ [3]. A global analysis revealed that four time constants are necessary for a sufficient description of both data sets. $\tau_{1}$ is somewhat ambiguous since it also contains coherent contributions of the solvent and is therefore not discussed any further.

PyA in MeOH shows a blue-shift of the excited state absorption (ESA, 620 to $600 \mathrm{~nm}$; Fig. 1, B and $\mathrm{C}$ ) and a corresponding red-shift of the stimulated emission (SE, 430 to $450 \mathrm{~nm}$ ) with a time constant $\tau_{2}$ of $10 \mathrm{ps}$ indicating a relaxation process on the $\mathrm{S}_{1}$ potential energy surface. A solvent with lower polarity e.g. $\mathrm{tBuOH}$, slows down this process from 10 ps to $155 \mathrm{ps}$. DMSO and all other aprotic solvents show a lack of this spectral shift of the ESA and SE, but exhibit a population which rises with a time constants $\tau_{2}$ in the same time range (12 ps in DMSO, $575 \mathrm{~nm}$ ). This leads to the conclusion that protic solvents interact with PyA via a specific hydrogen bond but only stabilize a state already existing in aprotic solvents. This state decays with $\tau_{3}(109 \mathrm{ps}$ in $\mathrm{MeOH}$ and $187 \mathrm{ps}$ in DMSO) to the final fluorescent state.

In aprotic solvents $\tau_{3}$ leads most likely to the shift of the SE around $450 \mathrm{~nm}$ which is the result of the faster decrease in intensity of this closely lying electronic state with an ESA at $575 \mathrm{~nm}$, which overlaps with the SE at early times. This state remains at this point not assignable, a TICT state as 
well as rotational diffusion were ruled out by quantum chemical calculations and magic angle measurements, respectively.

The remaining time constant $\tau_{4}(1.7 \mathrm{~ns}$ in $\mathrm{MeOH}$ and DMSO) describes the decay of the electronic state which dominates the overall spectra. The $\tau_{4}$ decay is dominated by the ESA signal (protic solvents: $\sim 600 \mathrm{~nm}$; aprotic solvents $\sim 650 \mathrm{~nm}$ ). The decay associated spectra indicate that PyA shows similar ultrafast dynamics in both classes of solvents but differ slightly in the reaction rates and stability of each electronic state.

\section{Discussion}

In accordance to other pyrene derivatives PyA reaches the $S_{1}$ state within our time resolution $\leq 200 \mathrm{fs}$ described by $\tau_{1}$. The $\tau_{2}$ and $\tau_{3}$ time constants cannot be assigned to specific electronic states, whereas $\tau_{4}$ definitely describes the repopulation of the ground state.

In protic solvents the spectral blue shift describes most likely a stabilization of the electronic potential surface on a time scale of approximately $10 \mathrm{ps}$ due to solvent interactions. This also indicates the existence of a hydrogen bond between PyA and the solvent since these processes are seen only in protic solvents. The process becomes more efficient with increasing solvent polarity as reflected by the overall faster time constants.

PyA in aprotic solvents exhibits the same time constants, but the spectra are dominated by the LE state absorption. Also the stabilized electronic state is depopulated on the same time scale (100 to $200 \mathrm{ps}$ ) and lies in the same spectral region as in protic solvents. The fact that PyA has a strong fluorescence as well as similar life times $(\sim 1.5 \mathrm{~ns})$ is in line with similar oscillator strengths obtained by DFT methods $\left(\mathrm{f}_{\text {osc }}\right.$ (unprotonated PyA $)=1.04$ and $\mathrm{f}_{\text {osc }}$ (protonated PyA $\left.)=1.05\right)$ for both solvent classes.

In summary, PyA fluorescence chracteristics are influenced by the solvent type and therefore by environmental changes, but these changes only weakly affect the overall fluorescence quantum yield. Introducing PyA into RNA structures therefore provides a direct observable for the structural (re-)arrangements and conformational changes of RNA with a high quantum yield fluorophore. To gain more insight into the molecular mechanisms of PyA photochemistry further DFT calculations will help to verify the energetically possible positions of the hydrogen bond and to explain the similarity of both species. An alternative approach for structural surveys will be tested by the linkage of adenosine at position 1 of pyrene which should show a weaker electronic coupling [10] and which possibly reduces the intercalation of PyA with the RNA. Currently other approaches like new chromophores and FRET-pairs as conformational probes for RNA are tested.

\section{References}

1. J. Huang, Y. Wu, Y. Chen, Z. Zhu, X. Yang, C. J. Yang, K. Wang, and W. Tan, Angew. Chem. Int. Ed., 50, 401-404 (2011).

2. N. Venkatesan, Y. J. Seo and B. H. Kim, Chem. Soc. Rev., 37, 648-663 (2008).

3. C. Wanninger-Weiß, L. Valis and H.-A. Wagenknecht, Bioorg. Med. Chem., 16, 100-106 (2008).

4. C. Grünewald, T. Kwon, N. Piton, U. Förster, J. Wachtveitl and J. Engels, Bioorg. Med. Chem. 16 (1), 1926 (2008).

5. U. Förster, K. Lommel, D. Sauter, C. Grünewald, J. W. Engels and J. Wachtveitl, ChemBioChem. 11, 664-672 (2010).

6. U. Förster, C. Grünewald, J. W. Engels and J. Wachtveitl, J. Phys. Chem. B 114, 11638-11645 (2010).

7. U. Förster, N. Gildenhoff, C. Grünewald, J. W. Engels and J. Wachtveitl, J. Lumin. 129, 1454-1458 (2009).

8. M. O. Lenz, R. Huber, B. Schmidt, P. Gilch, R. Kalmbach, M. Engelhard and J. Wachtveitl, Biophys. J. 91, 255-262 (2006).

9. S. A. Kovalenko, A. L. Dobryakov, J. Ruthmann and N. P. Ernsting, Phys. Rev. A 59, 2369-2384 (1999).

10. 10. A. G. Crawford, A. D. Dwyer, Z. Liu, A. Steffen, A. Beeby, L. Palsson, D. J. Tozer and T. B. Marder, J. Am. Chem. Soc. 133, 13349-13362 (2011). 